\title{
Response to the Comment on "Interaction of Two Fractions in a Degenerate Bose Gas at Finite Temperatures" (JETP Lett. 103, 403 (2016))
}

\author{
Yu. V. Likhanova ${ }^{a, b}$, S. B. Medvedev ${ }^{a}$, M. P. Fedoruk ${ }^{a, b}$, and P. L. Chapovsky ${ }^{b, c}$ \\ ${ }^{a}$ Institute of Computational Technologies, Siberian Branch, Russian Academy of Sciences, \\ pr. Akademika Lavrent'eva 6, Novosibirsk, 630090 Russia \\ ${ }^{b}$ Novosibirsk State University, ul. Pirogova 2, Novosibirsk, 630090 Russia \\ ${ }^{c}$ Institute of Automation and Electrometry, Siberian Branch, Russian Academy of Sciences, \\ pr. Akademika Koptyuga 1, Novosibirsk, 630090 Russia \\ e-mail: chapovsky@iae.nsk.su \\ Received May 20, 2016
}

DOI: $10.1134 / \mathrm{S} 0021364016120067$

The importance of studying the effects of interaction between the condensed and uncondensed fractions in a degenerate Bose gas was pointed out in the comment on our work [1]. We agree and mentioned the importance of such investigations ourselves.

The authors of the comment point out that the analysis of the experimental data should be conducted on the basis of a more complete theoretical model. The bibliography of such theoretical models was presented in [1]. However, the theoretical description of the expansion of a two-component Bose gas seems to be a very complicated problem. To give an example, we can mention the theory of an expanding Bose gas at finite temperatures proposed in [2]. Comparison of the theory [2] with three experimental studies of an expanding two-component gas [3-5] revealed contradictions between the experimental and theoretical results.

The comment also contains a number of statements that are difficult to agree with. The authors of the comment erroneously believe that our estimate of the accuracy (1.8\%) refers to the description of expansion of the condensed fraction in a two-component Bose gas. Our analysis consists in comparing the observed characteristics of two-component Bose gases with the Gross-Pitaevskii theory for pure condensates. In [1], the expansion of the pure condensed fraction was calculated, the accuracy of such calculations was estimated, comparison with the experiment was carried out, and quantitative conclusions about the interaction of two fractions were drawn.

It is worth mentioning that in [1] we solved the time-dependent Gross-Pitaevskii equation rather than the time-independent one, as the authors of the comment assume. The authors of the comment point out that, for the analysis of our experimental data, "much more useful would be the calculation of the steady-state density distributions of the normal and condensed phases by Eqs. (1)-(3)." However, the authors of the comment do not make any difference between the time-independent and time-dependent problems and it remains absolutely unclear how one should analyze the expansion of atoms, which is a time-dependent problem, on the basis of the calculation of steady-state distributions.

The authors of the comment point out that "such calculations were carried out many times and give much better agreement with the experiment (see, e.g. [6])." We could not find in [6] a solution of the timedependent problem of the expansion of a two-component gas we are interested in.

\section{REFERENCES}

1. Yu. V. Likhanova, S. B. Medvedev, M. P. Fedoruk, and P. L. Chapovsky, JETP Lett. 103, 403 (2016).

2. K. Gawryluk, M. Brewczyk, M. Gajda, and K. Rzazewski, J. Phys. B: At. Mol. Opt. Phys. 43, 105303 (2010).

3. F. Gerbier, J. H. Thywissen, S. Richard, M. Hugbart, P. Bouyer, and A. Aspect, Phys. Rev. A 70, 013607 (2004).

4. M. Zawada, R. Abdoul, J. Chwedenczuk, R. Gartman, J. Szczepkowski, L. Tracewski, M. Witkowski, and W. Gawlik, J. Phys. B: At. Mol. Opt. Phys. 41, 241001 (2008).

5. M. A. Caracanhas, J. A. Seman, E. R. F. Ramos, E. A. L. Henn, K. M. F. Magalhaes, K. Helmerson, and V. S. Bagnato, J. Phys. B: At. Mol. Opt. Phys. 42, 145304 (2009).

6. F. Dalfovo, S. Giorgini, L. P. Pitaevskii, and S. Stringari, Rev. Mod. Phys. 71, 463 (1999).

Translated by A. Safonov 